\title{
The Electromagnetic Wave Absorbing Property of Dual-Layer Cement Matrix Composites Based on the Principle of Electromagnetic Energy - Thermal Energy Conversion
}

\author{
Zuoqun Zhang, Chaoshan Yang*, Hua Cheng, Longlong Zhou, Junru Ren, Yuhao Zhu \\ Department of Civil Engineering, Army Logistics University of PLA, Chongqing 401311, China
}

Corresponding Author Email: YCSLQ13@126.com

https://doi.org/10.18280/ijht.390640

Received: 10 September 2021

Accepted: 15 November 2021

\section{Keywords:}

cement matrix electromagnetic wave (EMW) absorbing material, nanomaterial, wave absorbing agent, magnetic loss, dielectric loss

\begin{abstract}
Based on the application of stealth technology in actual engineering projects, and according to the theory that when electromagnetic waves (EMW) pass through different wave absorbing materials, they will undergo resistance loss, dielectric loss, and magnetic loss, and the electromagnetic energy will be converted into thermal energy, this paper designed and developed a dual-layer EMW-absorbing Cement Matrix Composites (CMC) combining with the principle of impedance matching, and proposed to take cement paste mixed with expanded perlite as the matching layer of the newly developed material, and aggregate and nanomaterials as its wave absorbing layer. The aggregate was prepared by mixing $15 \mathrm{wt} . \% 38 \mu \mathrm{m} \mathrm{Fe}_{3} \mathrm{O}_{4}$ powder and $10 \mathrm{wt} . \% 500 \mathrm{~nm} \mathrm{SiC}$ powder into the clay powder to produce cement raw pellets and calcine them at $1190^{\circ} \mathrm{C}$, the fabricated aggregate has obvious dielectric loss and magnetic loss to EMW, and its crushing resistance strength can reach $13.7 \mathrm{MPa}$. For the dual-layer test pieces made of the prepared aggregate and $2 \mathrm{wt} . \%$ Conductive Carbon Black (CCB), within 8-18GHz frequency band, the bandwidth with a reflectivity lower than $-10 \mathrm{db}$ reached $9.5 \mathrm{GHz}$, the bandwidth with a reflectivity lower than $-12 \mathrm{db}$ reached $7.55 \mathrm{GHz}$, and its maximum reflection loss reached $-19.56 \mathrm{db}$. In addition, the prepared CMC exhibited good mechanical properties, and its compressive strength reached $50.7 \mathrm{MPa}$ at the age of $28 \mathrm{~d}$.
\end{abstract}

\section{INTRODUCTION}

Cement is a widespread used substance in the construction of buildings, however, its ability to absorb EMW is quite limited. By incorporating wave-absorbing agents, composite porous aggregate, and wave-absorbing components, we could design and prepare the new-type CMC with multiple layers and novel surface structures. The EMW absorbing ability, and the ability to convert electromagnetic energy into thermal energy of the newly developed composite material have been improved, and the proposed material is going to exert its effectiveness in fields such as microwave deicing, and electromagnetic pollution control, prevention, and treatment.

The wave-absorbing performance of a composite material largely depends on its loss of EMW energy, and its essence is to convert electromagnetic energy into thermal energy and consume it. According to different wave-absorbing mechanisms, it could be divided into resistive loss, magnetic loss, and dielectric loss [1]. Resistive lossy materials such as carbon black and graphite generally have the characteristics of low resistivity and good conductivity. When conductive materials are added into cement matrix materials, the conductive materials will contact each other and a conductive network will be formed inside the materials. The conductive materials in alternating electromagnetic field will make use of their resistance characteristics and convert the electromagnetic energy into thermal energy, thereby realizing the purpose of absorbing the electromagnetic energy. Usually, the addition amount of such microwave absorbing agent in cement matrix materials is relatively small, this is because an excessive amount can strengthen the reflection of EMW on the material surface and reduce the microwave absorbing performance of the sample. The dielectric loss of materials such as $\mathrm{TiO}_{2}$ and $\mathrm{MnO}_{2}$ refers to the process in which the wave-absorbing agent generates a local conductive network in the alternating electric field, forms oscillating current, consumes part of the electromagnetic energy, and converts it into heat energy. The magnetic loss refers to the process in which the waveabsorbing agent undergoes irreversible magnetization due to the action of external magnetic field, and then forms magnetic hysteresis, eddy current, magnetic resonance, and other losses, and converts part of the electromagnetic energy into the heat energy; ferrite materials are the most studied magnetic loss materials in existing research. The materials incorporated into cement base as wave-absorbing agents can be roughly divided into: micro-powders, conductive fibers, and new-type waveabsorbing agents, etc. Wherein, micro-powders include carbon-based materials (such as multi-walled carbon nanotubes, $\mathrm{CCB}$, and graphite powder), $\mathrm{TiO}_{2}$ micro-powder, $\mathrm{MnO}_{2}$ micro-powder, and ferrite micro-powder, etc. [2]. Dai et al. [3] developed a CMC by mixing carbon black with different mass fractions, the prepared material exhibited broadband absorption and high strength performance. Studies suggest that adding carbon black can significantly improve the permittivity of cement materials, and carbon black concrete shows good EMW-absorbing performance in the frequency range of 8-26.5 GHz. When the mass content of carbon black is $2.5 \%$, the frequency bandwidth of $\mathrm{CMC}$ with a reflectivity lower than $-10 \mathrm{~dB}$ is $14.9-26.5 \mathrm{GHz}$, and its compressive strength reaches $50 \mathrm{MPa}$. Qiu et al. [4] studied the EMW 
absorbing property of CMC mixed with high-purity graphite powder, and their research results showed that in the $\mathrm{S}$ frequency band, the CMC mixed with $15 \%$ graphite by mass fraction had a higher permittivity; when the thickness of the wave absorbing layer was $5 \mathrm{~mm}$, the effective absorption frequency band with a reflectivity less than $-10 \mathrm{~dB}$ was relatively narrow, only $0.43 \mathrm{GHz}(3.62-3.95 \mathrm{GHz})$, and at the same time, the graphite particle size would affect the wave absorbing ability of the material. Lv et al. [5] mixed ferrite and graphite into the cement base at the same time to study its microwave absorbing property, and the orthogonal experiment results showed that, the amount of micro ferrite affected the absorption width within $0.5-4 \mathrm{GHz}$, while the amount of graphite affected the absorption depth of the material. Within this frequency range, adding graphite can improve the spatial wave impedance. Carbon nanotubes have large aspect ratio and specific surface area, and good electrical conductivity, which meet the requirements of wave absorbing materials in terms of strong absorption performance and low density, they can attenuate EMW through induced current formed by the resonance oscillation of the pole. At the same time, carbon nanotubes and the hydration products of cement can combine to form a network-shaped conductive structure. When EMW are incident and generate vortex-shaped induced currents, they can consume the energy of EMW through the heat loss of resistance [6]. Wang et al. [2] made a Carbon Nano Tube (CNT) suspension liquid with surfactant-ultrasonic treatment, in this study, the multi-walled CNT were incorporated into cement matrix to fabricate composites and study its mechanical and wave-absorbing properties; his research results showed that, silica fume can help carbon nanomaterials disperse uniformly in cement and enhance the mechanical strength of the test pieces. When mixed with $0.6 \mathrm{wt} \%$ multiwalled carbon nanotubes and $8 \mathrm{wt} \%$ rubber, the bandwidth of CMC with a reflectivity less than $-10 \mathrm{~dB}$ reached $4.7 \mathrm{GHz}$ and the minimum reflectivity reached $-13.6 \mathrm{~dB}$. Xiong et al. [7] studied the microwave absorbing property of CMC respectively made of nano-titanium oxide, nickel oxide, carbonyl iron powder, and ordinary Portland cement; and their research results indicated that, within $8-18 \mathrm{GHz}$ frequency band, the CMC mixed with nano-titanium oxide reached the minimum reflectivity of $-16.34 \mathrm{~dB}$ at $16.24 \mathrm{GHz}$, its bandwidth with a reflectivity less than $-10 \mathrm{~dB}$ was $4.5 \mathrm{GHz}$, and the reflectivity was always less than $-7 \mathrm{~dB}$. Guo et al. [8] used ferrite or graphite to coat fly ash and closed-pore perlite to fabricate resonator, which was then mixed into cement base and its wave-absorbing performance was tested; the test results showed that the reflectivity was less than $-10 \mathrm{~dB}$ within the 9$18 \mathrm{GHz}$ frequency band. Since the coating method is easy to operate and the material has good wave absorbing performance, the closed-pore perlite cement-matrix waveabsorbing composites has certain advantages in engineering applications.

This study designed a dual-layer CMC incorporated by aggregate and different nanomaterials, respectively studied the surface and internal micro-morphology, electromagnetic parameter performance, and crushing resistance strength of the aggregate, and the reflection loss and compressive strength of the CMC test boards. The dual-layer CMC designed in this paper can be used for the construction of exterior walls, and it can provide structural support and good electromagnetic protection.

\section{EXPERIMENT}

\subsection{Raw materials}

Experimental materials include: 1) 325 mesh clay powder product of Changzhou Dingbang Mineral Products Technology Co., Ltd., acted as aggregate matrix in the experiment; 2) $38 \mu \mathrm{m} \mathrm{Fe}_{3} \mathrm{O}_{4}$ of $99.99 \%$ purity product of Hebei Hangbei Metal Material Co., Ltd., acted as precursor of absorption phase in the experiment; 3) $500 \mathrm{~nm}$ SiC produced by Taipeng Metal Materials Co., Ltd., also acted as precursor of absorption phase in the experiment; 4) Multi-walled carbon nanotubes of $99 \%$ purity product of Shenzhen Suiheng Technology Co., Ltd., acted as wave absorbing agent in the experiment, its structure is shown in Table 1; 5) Super $\mathrm{P}$ Li CCB powder (product of TIMCAL), acted as wave absorbing agent in the experiment, its parameters are shown in Table 2; 6) $\mathrm{TiO}_{2}$ with a particle size of $20 \mathrm{~nm}$ product of Hebei Chuancheng Metal Material Co., Ltd. acted as wave absorbing agent in the experiment; 7) Fly ash product of Henan Boruntao Material Co., Ltd., used in the experiment to improve the workability and chemical resistance of the material; 8) PO 42.5 Portland cement conforms to GB 175-2007; 9) Quartz sand with a fineness modulus of 2.9, acted as fine aggregate in the experiment; 10) Expanded perlite with an apparent density of $120 \mathrm{~kg} / \mathrm{m}^{3}$ and an average diameter of $1.1 \mathrm{~mm}$ product of Baichuan Mining Co., Ltd., Pingqiao District, Xinyang City, acted as the matching layer substance in the experiment.

Table 1. Structural parameters of the multi-walled carbon nanotubes

\begin{tabular}{ccc}
\hline Parameter & Value & $\begin{array}{c}\text { Measuring } \\
\text { method }\end{array}$ \\
\hline $\begin{array}{c}\text { Carbon tube length }(\mu \mathrm{m}) \\
\text { Carbon tube outer diameter } \\
(\mathrm{nm})\end{array}$ & $3 \sim 12$ & TEM \\
$\begin{array}{c}\text { Carbon tube wall thickness } \\
(\mathrm{nm})\end{array}$ & $4.1 \sim 5.4$ & HRTEM \\
$\begin{array}{c}\text { Number of carbon tube layers } \\
\text { Length to diameter ratio (LD) }\end{array}$ & $8 \sim 16.4$ & HRTEM \\
& & TEM \\
\hline
\end{tabular}

Table 2. Parameters of CCB

\begin{tabular}{ccccc}
\hline $\begin{array}{c}\text { Grade of } \\
\text { the } \\
\text { carbon } \\
\text { black }\end{array}$ & $\begin{array}{c}\text { Particle } \\
\text { size } \\
(\mathbf{n m})\end{array}$ & $\begin{array}{c}\text { Oil } \\
\text { absorption } \\
(\mathbf{m l} / \mathbf{1 0 0 g})\end{array}$ & $\begin{array}{c}\text { Ash } \\
\text { content \% }\end{array}$ & $\begin{array}{c}\text { BET } \\
\text { specific } \\
\text { surface } \\
\text { area }\end{array}$ \\
\hline $\begin{array}{c}\text { SUPER P } \\
\text { LI }\end{array}$ & 40 & 290 & 0.01 & 62 \\
\hline
\end{tabular}

\subsection{Sample preparation}

(1) Preparation of aggregate: $75 \mathrm{wt} . \%$ clay powder, $15 \mathrm{wt} . \%$ $38 \mu \mathrm{m} \mathrm{Fe}_{3} \mathrm{O}_{4}$, and $10 \mathrm{wt} . \% 500 \mathrm{~nm} \mathrm{SiC}$ were weighed and put into the Model GMS-30-4 drum-type mill pot to roll and mix for 15 minute at a speed of $200 \mathrm{r} / \mathrm{min}$. Then, the uniformly mixed materials were placed into an 80-type disc-shaped granulator, a water sprayer was used to spray water intermittently to the dry materials being granulated in the granulator, and a sieve was used to screen out aggregate with a diameter between $8-12 \mathrm{~mm}$. After that, the prepared aggregate was dried to constant weight at $105^{\circ} \mathrm{C}$, calcined in a 
muffle furnace with heating rate $10^{\circ} \mathrm{C} \cdot \mathrm{min}^{-1}$, held at $600^{\circ} \mathrm{C}$ for 8 minutes, and then heated continuously to $1190^{\circ} \mathrm{C}$ and kept for $1 \mathrm{~h}$. At last, the aggregate was chilled to $130^{\circ} \mathrm{C}$ in the cavity with cooling rate $8^{\circ} \mathrm{C} \cdot \mathrm{min}^{-1}$, then fetched out and naturally chilled to room temperature. By this means, the aggregate with EMW absorbing ability was prepared (see Figure 1).

(2) Preparation of test pieces for reflectivity and compression tests: Table 3 gives the material ratios of different test pieces. The calcined aggregate was mixed with different nanomaterials to prepare the wave absorbing layer of CMC. The volume ratio of aggregate and cement paste was controlled at 45\%:55\%. Cement, sand, fly ash, and water (mass ratio 1:1.1:0.1:0.32) were mixed and stirred for $1 \mathrm{~min}$ using a stirrer, and then nanomaterials that were subjected to dispersion pre-treatment were added and mixed for 2 min. After that, the mixture was placed into a steel mold sized $180 \mathrm{~mm} \times 180 \mathrm{~mm} \times 40 \mathrm{~mm}$ until reached $30 \mathrm{~mm}$ height, and shaken on a vibrating table for $1 \mathrm{~min}$. After the wave absorbing layer was initially set for about 1 hour, substance of the matching layer (thickness $10 \mathrm{~mm}$ ) was poured on top of the wave absorbing layer just prepared.

The matching layer substance was mortar. Cement paste: expanded perlite was 40\%:60\% in volume ratio, water: cement was 0.32 in mass ratio. The CMC was used to prepare test pieces sized $150 \mathrm{~mm} \times 150 \mathrm{~mm} \times 150 \mathrm{~mm}$ for the compression test. At the same time, two-layer test pieces of CMC mixed with ordinary clay ceramsite aggregate and crushed stone aggregate were prepared. Their mixing ratios were the same with that of the $\mathrm{CMC}$, and the ranges of the particle size of crushed stone and clay ceramsite were both within $8-12 \mathrm{~mm}$. All test pieces were cured for $24 \mathrm{~h}$ in a standard curing room with a temperature of $20^{\circ} \mathrm{C} \pm 2^{\circ} \mathrm{C}$ and a relative humidity of $95 \%$, and then demolded and kept in the standard curing lab for solidification. The test pieces for the reflectivity test were taken out for testing after 28 days of curing.

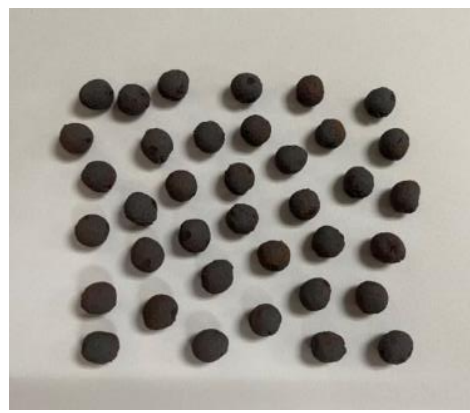

Figure 1. The self-prepared aggregate

Table 3. Material ratios of test pieces

\begin{tabular}{ccccc}
\hline Test piece & Aggregate type & Type and content of absorbing agent (wt.\%) & Matching layer & Size of test piece \\
\hline Q1 & Crushed stone & No & Yes & $180 \mathrm{~mm} \times 180 \mathrm{~mm} \times 40 \mathrm{~mm}$ \\
Q2 & Clay ceramsite & No & Yes & $180 \mathrm{~mm} \times 180 \mathrm{~mm} \times 40 \mathrm{~mm}$ \\
Q3 & Functional aggregate & $2 \%$ multi-walled carbon nanotubes & Yes & $180 \mathrm{~mm} \times 180 \mathrm{~mm} \times 40 \mathrm{~mm}$ \\
Q4 & Functional aggregate & $4 \%$ multi-walled carbon nanotubes & Yes & $180 \mathrm{~mm} \times 180 \mathrm{~mm} \times 40 \mathrm{~mm}$ \\
Q5 & Functional aggregate & $6 \%$ multi-walled carbon nanotubes & Yes & $180 \mathrm{~mm} \times 180 \mathrm{~mm} \times 40 \mathrm{~mm}$ \\
Q6 & Functional aggregate & $2 \%$ Super P Li CCB & No & $180 \mathrm{~mm} \times 180 \mathrm{~mm} \times 30 \mathrm{~mm}$ \\
Q7 & Functional aggregate & $2 \%$ Super P Li CCB & Yes & $180 \mathrm{~mm} \times 180 \mathrm{~mm} \times 40 \mathrm{~mm}$ \\
Q8 & Functional aggregate & $3 \%$ Super P Li CCB & Yes & $180 \mathrm{~mm} \times 180 \mathrm{~mm} \times 40 \mathrm{~mm}$ \\
Q9 & Functional aggregate & $4 \%$ Super P Li CCB & Yes & $180 \mathrm{~mm} \times 180 \mathrm{~mm} \times 40 \mathrm{~mm}$ \\
Q10 & Functional aggregate & $3 \%$ nano TiO & Yes & $180 \mathrm{~mm} \times 180 \mathrm{~mm} \times 40 \mathrm{~mm}$ \\
Q11 & Functional aggregate & $5 \%$ nano TiO 2 & Yes & $180 \mathrm{~mm} \times 180 \mathrm{~mm} \times 40 \mathrm{~mm}$ \\
Q12 & Functional aggregate & $7 \%$ nano TiO & Yes & $180 \mathrm{~mm} \times 180 \mathrm{~mm} \times 40 \mathrm{~mm}$ \\
Q13 & Functional aggregate & $2 \%$ Super P Li CCB & No & $150 \mathrm{~mm} \times 150 \mathrm{~mm} \times 150 \mathrm{~mm}$ \\
Q14 & Functional aggregate & $3 \%$ Super P Li CCB & No & $150 \mathrm{~mm} \times 150 \mathrm{~mm} \times 150 \mathrm{~mm}$ \\
Q15 & Functional aggregate & $4 \%$ Super P Li CCB & No & $150 \mathrm{~mm} \times 150 \mathrm{~mm} \times 150 \mathrm{~mm}$ \\
\hline
\end{tabular}

\subsection{Test method}

SEM (Scanning electron microscope) was adopted to scan the surface and cross profile morphology of the self-prepared aggregate to survey its external and internal structures after calcination. The Quanta TM 250FEG field emission SEM and EDS (Energy Dispersive spectrometer) used in this research were products of American company FEI, and the maximum resolution could reach $0.8 \mathrm{~nm}$ under high vacuum conditions.

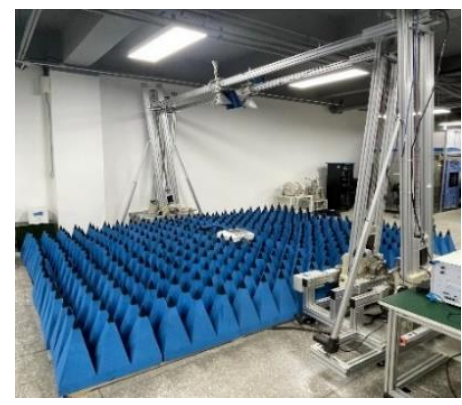

Figure 2. Test equipment for the arch method
If the tangent of loss angle and the imaginary part of electromagnetic parameter of the target are relatively large, then the material has good EMW absorbing performance. Rectangular waveguide method and coaxial transmission line method are two commonly used methods for testing electromagnetic parameters. This paper adopted the rectangular waveguide method to test the electromagnetic parameters of calcined aggregates of different material ratios. Water was added into the dry raw materials incorporated with aggregate and then the composites were made into rectangular-shaped test pieces and calcined using the same method with the aggregate, and then cooled and polished into standard test pieces with a 3L SERIES V800 machine tool. The size of the test pieces for the rectangular waveguide method was: for the $8-12 \mathrm{GHz}$ frequency band, the size was $22.86 \times 10.16 \times 2.5 \mathrm{~mm}$; for the $12-18 \mathrm{GHz}$ frequency band, the size was $15.8 \times 7.9 \times 2.5 \mathrm{~mm}$. After that, the test pieces were fixed by a standard clamp on the vector network analyzer (Model E8363B produced by Agilent) and measured for complex permeability and complex permittivity. According to the GJB 2038-1994 Methods for Measurement of Reflectivity of Radar Absorbing Material, samples of the CMC were made 
into test boards sizes $180 \times 180 \times 30 / 40 \mathrm{~mm}$, and the arch method was adopted to measure the reflectivity; the test frequency band was $8-12 \mathrm{GHz}$, Figure 2 shows a photo of the equipment. The crushing resistance strength of the aggregate and the compressive strength of CMC were measured on the MTS2000 test system.

\section{RESULTS AND DISCUSSION}

\subsection{Microstructure analysis of the aggregate}

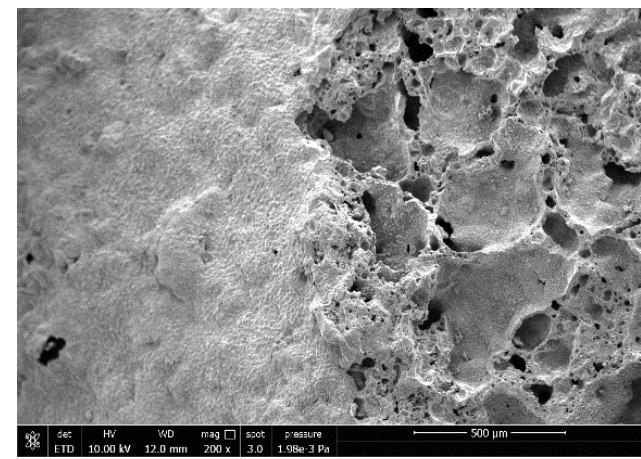

(a) $\times 200$

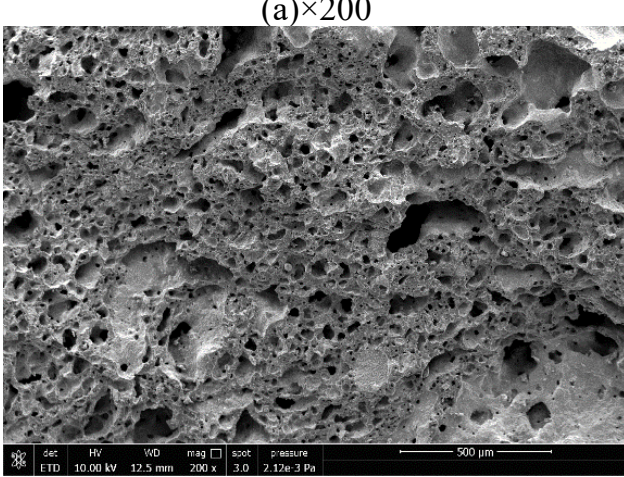

(b) $\times 200$

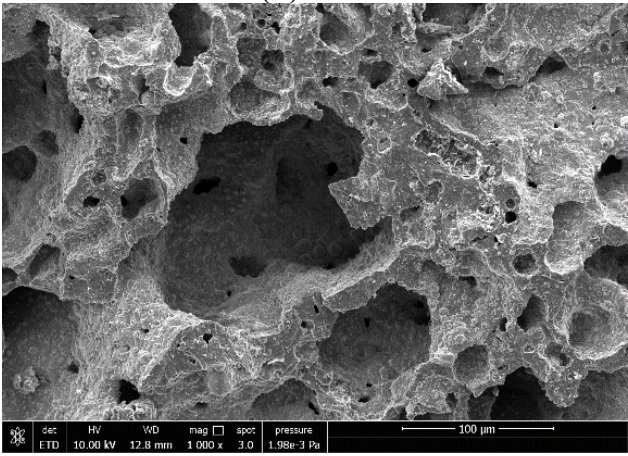

(c) $\times 1000$

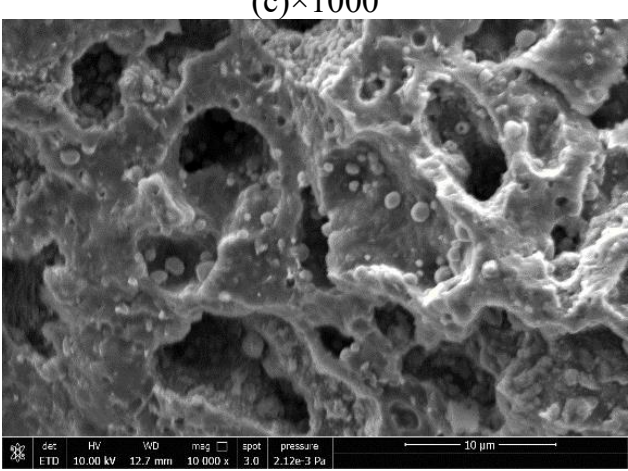

(d) $\times 10000$

Figure 3. SEM photos of the aggregate (a is the external and internal interface; b, c, d are internal structures)
Image (a) in Figure 3 shows the SEM photos of external and internal interface of the prepared aggregate. The surface of raw pellets was glazed during high-temperature calcination and turned smoother, which had ensured the strength of the aggregate. Images (b) and (c) in Figure 3 are SEM photos of the cross profile of the aggregate. During decomposition at high temperature, carbonate, sulfate, and other matters in clay powder will generate lots of closed pores and connected pore canals, which will enhance the resonance loss of aggregate to EMW. Image (d) in Figure 3 shows that $\mathrm{Fe}_{2} \mathrm{O}_{3}$ (white particles) uniformly embedded in the aggregate, which can ensure the microwave absorbing performance of the aggregate.

\subsection{Analysis of electromagnetic parameters of aggregate}

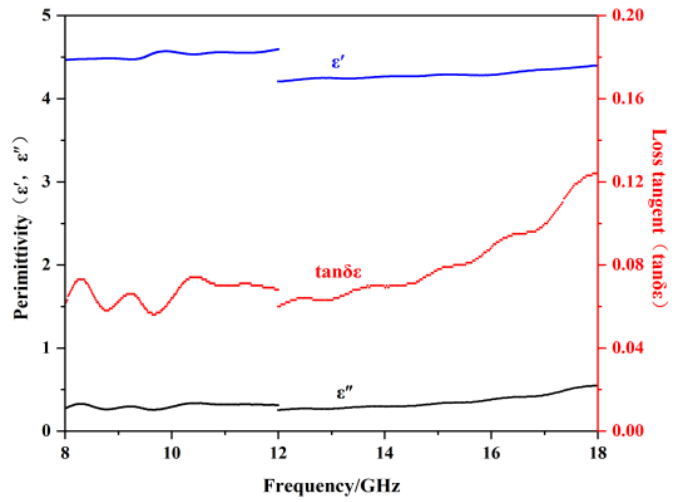

(a) Permittivity of the aggregate

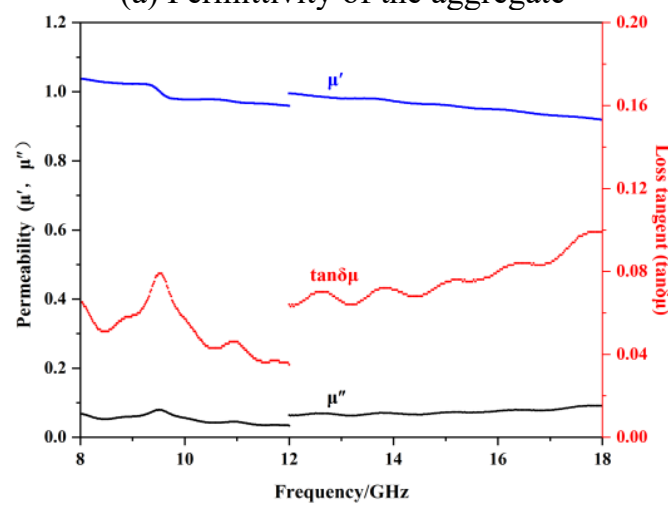

(b) Magnetic permeability of the aggregate

Figure 4. Electromagnetic parameters of the aggregate calcined at $1190^{\circ} \mathrm{C}$

Figure 4 gives the measurement results of the electromagnetic parameters of aggregate calcined at $1190^{\circ} \mathrm{C}$. The electromagnetic loss ability of the material is directly related to the imaginary part of the parameter and the tangent of loss angle. In $8-18 \mathrm{GHz}$ test frequency band, the real part $\varepsilon^{\prime}$ of the permittivity was within 4.21-4.60, and the imaginary part $\varepsilon^{\prime \prime}$ was within $0.252-0.546$. The tangent angle $\tan \delta \varepsilon$ of permittivity was within $0.06-0.12$. When frequency changed, the tangent of electrical loss angle did not change significantly, and the tangent value of electrical loss angle was relatively large, indicating that the aggregate had certain dielectric loss ability. The real part $\mu^{\prime}$ of permeability was between 0.92 and 1.04 , the imaginary part $\mu^{\prime \prime}$ was between 0.043 and 0.091 , and the tangent angle $\tan \delta \mu$ of permeability was between 0.04 and 0.10 . Within test frequency band, the large imaginary part of magnetic permeability and tangent value of magnetic loss angle were closely associated with the wave absorbing agents $\mathrm{SiC}$ and $\mathrm{Fe}_{3} \mathrm{O}_{4}$, indicating that the aggregate had good magnetic loss performance. 


\subsection{Analysis of the wave absorbing performance of CMC}

The design of CMC test boards for reflectivity test is listed in Table 3; and the reflection loss of test pieces are plotted in Figures 5(a)-(f). Under identical experimental conditions, less reflection loss indicates stronger EMW loss ability of the material. Figure 5(a) suggests the reflection loss of test piece Q1 made of crushed stone aggregate was $-3.81 \sim-5.35 \mathrm{~dB}$ within $8-18 \mathrm{GHz}$ frequency band, as for test piece Q2 made of clay ceramsite aggregate, the value of its reflection loss of was $-5.28 \sim-6.47 \mathrm{~dB}$, indicating that EMW absorption of composites containing clay ceramsite was better than that containing gravel concrete. The resonant cavity formed by the large number of pores in clay ceramsite acted positively on the absorption of EMW under the resonance absorption effect, but the EMW absorbing ability of the two test boards was low, which cannot meet the requirements of electromagnetic protection for broad band and strong absorption. Figure 5(b) shows the reflection loss of test pieces with different contents of multi-walled carbon nanotubes. For test pieces Q3, Q4 and Q5, their reflection loss values were respectively -8.98 $15.36 \mathrm{~dB},-8.41 \sim-15.07 \mathrm{~dB}$ and $-7.65 \sim-10.75 \mathrm{~dB}$. For the duallayer test piece Q3 (mixed with aggregate and 2 wt.\% multiwalled carbon nanotubes), within the frequency band range of $8-18 \mathrm{GHz}$, the bandwidth with a reflectivity less than $-10 \mathrm{db}$ reached $7.85 \mathrm{GHz}$. With the increase of the content of multiwalled carbon nanotubes, the wave absorbing performance of the CMC decreased accordingly. Figure 5(c) shows the reflection loss values of single-layer and dual-layer CMC, and the EMW absorbing ability of dual-layer test piece had been greatly improved. The impedance matching of multi-layer materials means that the wave impedance between free space and medium, or between different medium layers reaches good matching, so that EMW can enter the outermost medium layer from the air medium as much as possible, then after attenuated by the wave absorbing medium, the EMW can enter the next medium layer with less reflection, in this way, the process of EMW absorption was completed. This study added expanded perlite into the matching layer, expanded perlite is a porous material with a honeycomb-shaped internal structure and low electromagnetic parameters, and it can effectively decrease the permittivity of the substance, while reducing the impedance difference between air and CMC, it also reduces the interface reflection at the same time, and improves the impedance matching performance of the CMC, which is good for the absorption of EMW by the wave absorbing layer. The CMC mixed with $\mathrm{CCB}$ is a kind of electrical loss medium. The incorporation of Super P Li CCB increases the relative loss factor of the composite material, and EMW are attenuated through ion polarization, electronic polarization, and interface polarization. At the same time, when EMW pass through the composite material, the eddy currents generated inside would convert electromagnetic energy into heat energy, which will dissipate later $[9,10]$. Figure 5(d) shows the reflection loss of test pieces containing varying Super P Li CCB content. The reflection loss values of Q7, Q8 and Q9 were respectively $9.79 \sim-19.56 \mathrm{~dB},-8 \sim-11.69 \mathrm{~dB}$, and $-7.23 \sim-10.88 \mathrm{~dB}$. For the dual-layer test piece Q7 (mixed with aggregate and 2wt.\% Super P Li CCB), within $8-18 \mathrm{GHz}$ frequency band, the bandwidth with a reflectivity less than $-10 \mathrm{db}$ reached $9.5 \mathrm{GHz}$, and the bandwidth with a reflectivity less than $-12 \mathrm{db}$ reached 7.55 GHz. As the content of Super P Li CCB increases, the wave-absorbing performance of the $\mathrm{CMC}$ decreases. Figure 5 (e) shows the reflection loss of test pieces with varying contents of nano- $\mathrm{TiO}_{2}$, the reflection loss value of $\mathrm{Q} 10$ was 8.65 11.33dB, Q11 and Q12 both showed multiple obvious absorption peaks, and their reflection loss values were $8.73 \sim 16.17 \mathrm{~dB}$ and $-5.62 \sim 15.65 \mathrm{~dB}$, respectively. For the duallayer test piece Q10 (mixed with aggregate and $3 \mathrm{wt} . \%$ nano $\mathrm{TiO}_{2}$ ), within the frequency band range of $8-18 \mathrm{GHz}$, the bandwidth with a reflectivity less than $-10 \mathrm{db}$ reached $8.05 \mathrm{GHz}$. As for test piece Q11 (mixed with aggregate and $5 \mathrm{wt} . \%$ nano $\mathrm{TiO}_{2}$ ), within the frequency band range of $8-18 \mathrm{GHz}$, the bandwidth with a reflectivity less than $-10 \mathrm{db}$ reached 8.25 $\mathrm{GHz}$, and the bandwidth with a reflectivity less than $-12 \mathrm{db}$ reached 5.6GHz. In terms of test piece Q12 (mixed with aggregate and $7 \mathrm{wt} . \%$ nano $\mathrm{TiO}_{2}$ ), within the frequency band range of $8-18 \mathrm{GHz}$, the bandwidth with a reflectivity less than $-10 \mathrm{db}$ reached $6.1 \mathrm{GHz}$. Due to the small particle size of nano $\mathrm{TiO}_{2}$, the quantum size effect can cause energy level splitting in $\mathrm{TiO}_{2}$, creating new wave absorption channels. At the same time, the imaginary part of electromagnetic parameters of nano $\mathrm{TiO}_{2}$ is relatively large, and the EMW are absorbed through dielectric loss. Due to the different particle sizes of composition materials of the CMC, it's difficult to achieve uniform distribution of components through the stirring of stirrer. This paper compared the reflection loss of test piece Q11 twice. Figure 5(f) gives a comparison graph of the reflection loss of Q11 before and after rotating $90^{\circ}$. As shown in the figure, the trends of reflection loss to the two tests were the same, and the value difference was not much, indicating that the $\mathrm{CMC}$ prepared in this study was relatively uniform.

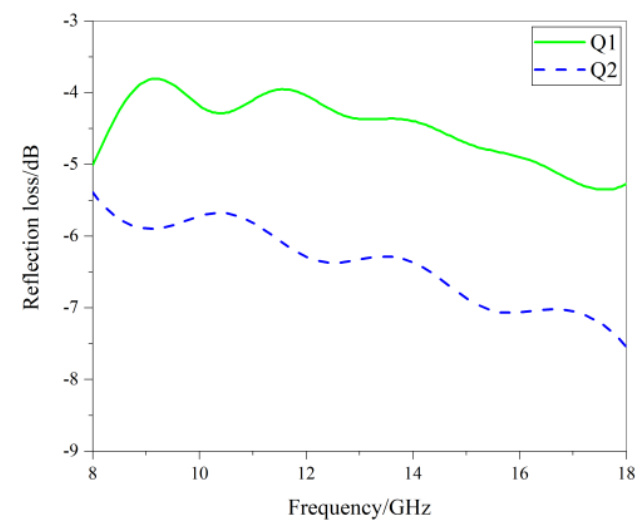

(a) Reflection loss of test pieces incorporated with $8-12 \mathrm{~mm}$ diameter gravel and clay ceramsite aggregate

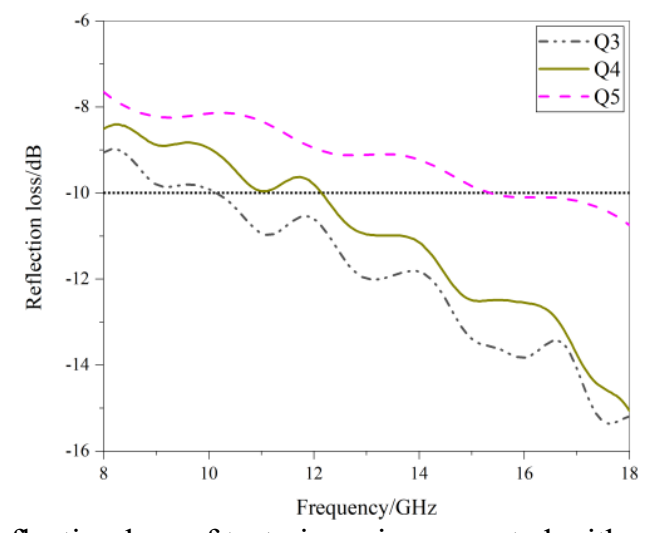

(b) Reflection loss of test pieces incorporated with varying contents of multi-walled carbon nanotubes 


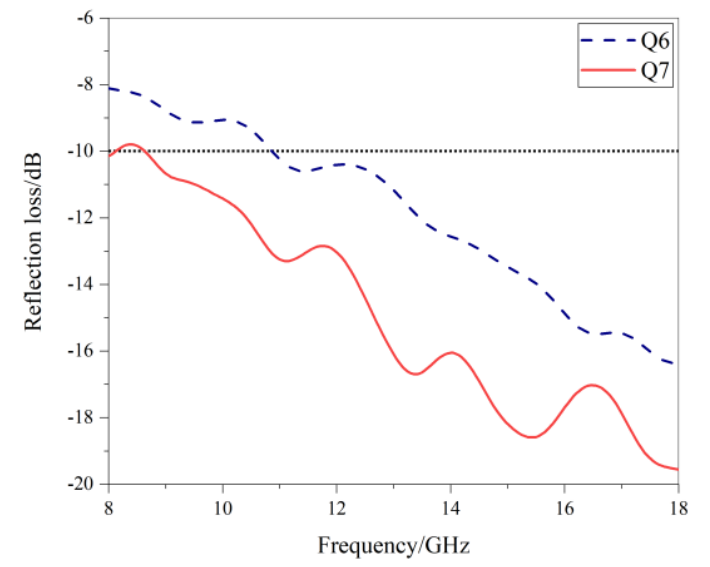

(c) Reflection loss of single-layer and dual-layer test pieces

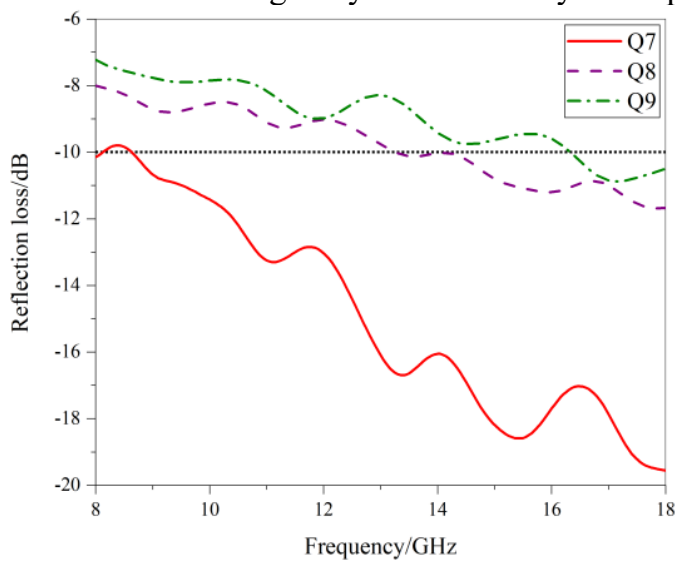

(d) Reflection loss of test pieces incorporated with varying contents of Super P Li CCB

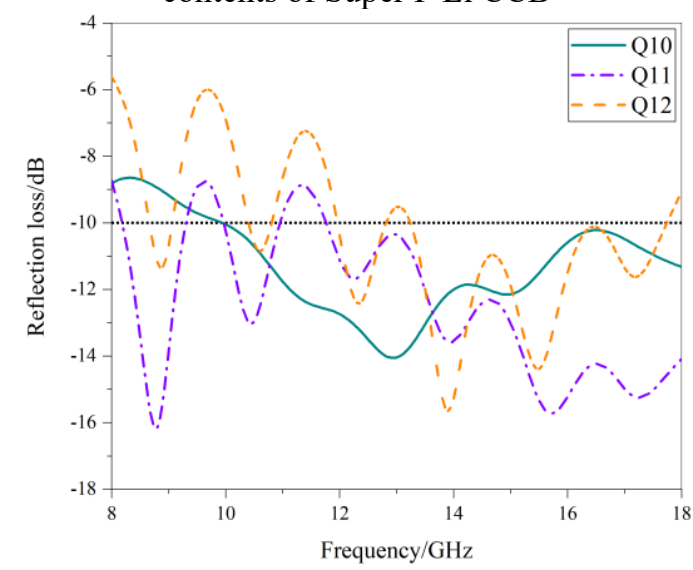

(e) Reflection loss of test pieces incorporated with varying contents of nano- $\mathrm{TiO}_{2}$

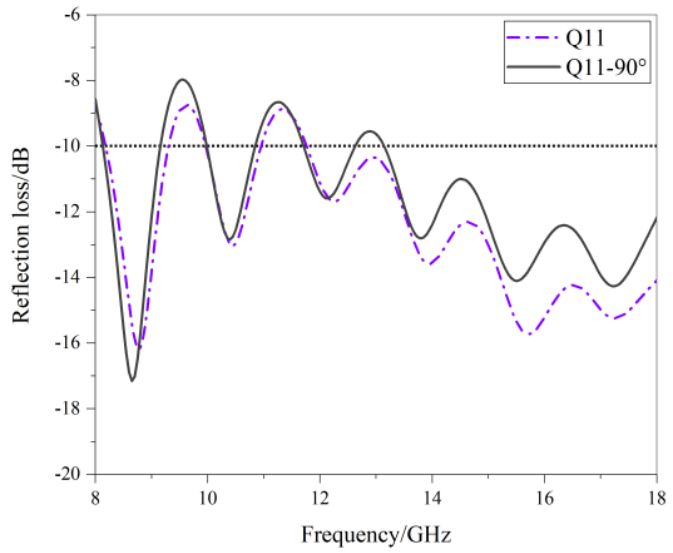

(f) Comparison of reflection loss of test piece under a same reflectivity before and after rotating $90^{\circ}$

Figure 5. Reflection loss of test pieces

\subsection{Analysis of mechanical properties of aggregate and CMC}

Aggregate accounted for about 45\% (in volume) of the wave-absorbing layer of $\mathrm{CMC}$. The crushing resistance strength of the prepared aggregate reached $13.7 \mathrm{MPa}$, which played a positive role in improving the compressive performance of CMC. Under identical mixing ratio and curing conditions, the compressive strength test data of the wave absorbing layer of CMC mixed with different contents of Super P Li CCB are shown in Figure 6. The compressive strength of different test pieces was Q14 $>$ Q13 $>$ Q15 $=46.9 \mathrm{MPa}$, therefore, the test pieces mixed with aggregate and Super $\mathrm{P} \mathrm{Li}$ CCB generally had high compressive strength. The CCB material with a particle size of $40 \mathrm{~nm}$ can fill the pores formed after the hydration of CMC and improve the grading of CMC. $\mathrm{CCB}$ has good water retention property and can promote cement hydration by enhancing the ability of the composite material to retain moisture [10]. However, when the content of CCB increases gradually, the CCB particles will adhere to the surface of cement particles, hindering the contact between water and cement particles, thereby affecting the hydration of cement, and resulting in a decrease in the compressive strength of CMC. In this study, for test piece Q14 (mixed with aggregate and 3wt.\% Super $\mathrm{P}$ Li CCB), its compressive strength on $28 \mathrm{~d}$ reached $52.6 \mathrm{MPa}$, indicating that the test piece had excellent mechanical properties.

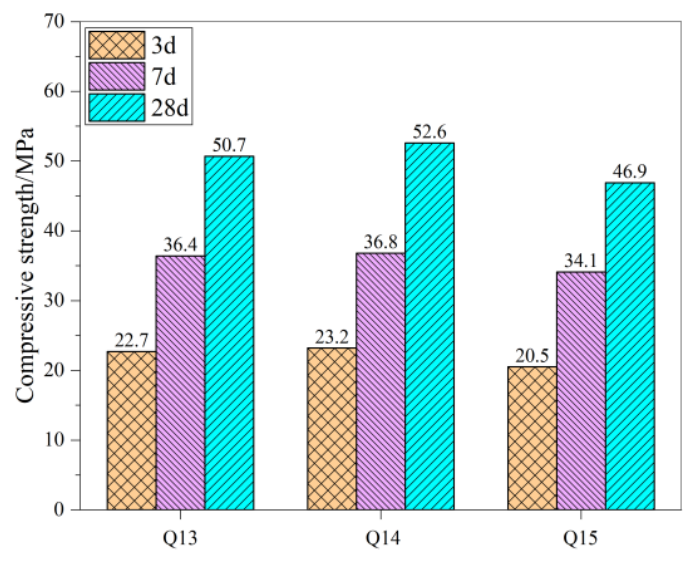

Figure 6. Compressive strength of the wave-absorbing layer of test pieces incorporated with varying contents of Super $\mathrm{P}$ $\mathrm{Li}$ CCB

\section{CONCLUSION}

In summary, by mixing nano $\mathrm{SiC}$ and $\mathrm{Fe}_{3} \mathrm{O}_{4}$ into clay powder, using disc granulator to make raw pellets, and calcinating at $1190^{\circ} \mathrm{C}$, this study prepared a kind of aggregate with internal porous structure and good dielectric loss and magnetic loss properties, and its crushing resistance strength reached $13.7 \mathrm{MPa}$. The incorporation of nanomaterials can improve the wave absorbing performance of $\mathrm{CMC}$, and the effect is more significant at high frequencies. For the duallayer test pieces mixed with aggregate and $2 \mathrm{wt} . \%$ multi-walled carbon nanotubes, within $8-18 \mathrm{GHz}$ frequency band, the bandwidth with a reflectivity less than $-10 \mathrm{db}$ (meaning more than $90 \%$ EM energy absorption) reached $7.85 \mathrm{GHz}$. For duallayer test pieces mixed with aggregate and 2 wt.\% Super P Li $\mathrm{CCB}$, within the frequency band range of $8-18 \mathrm{GHz}$, the bandwidth with a reflectivity less than $-10 \mathrm{db}$ reached $9.5 \mathrm{GHz}$, 
the bandwidth with a reflectivity less than $-12 \mathrm{db}$ reached 7.55 $\mathrm{GHz}$ (corresponding to greater than 93.7\% EM energy absorption), and the minimum reflectivity was $-19.56 \mathrm{db}$. As for test pieces mixed with aggregate and 5 wt.\% nano- $\mathrm{TiO}_{2}$, within the frequency band range of $8-18 \mathrm{GHz}$, the bandwidth with a reflectivity less than $-10 \mathrm{db}$ reached $8.25 \mathrm{GHz}$, and the bandwidth with a reflectivity less than $-12 \mathrm{db}$ reached $5.6 \mathrm{GHz}$, their ability to absorb EMW was good. Moreover, the compressive strength of CMC mixed with aggregate and 3 wt.\% Super P Li CCB reached $50.7 \mathrm{MPa}$ at the age of 28 days, which had met the strength requirements of ordinary building components.

\section{ACKNOWLEDGMENT}

Funded by the Chongqing Postgraduate Research and Innovation Project (Grant No.: CYS21530).

\section{REFERENCES}

[1] Wu, J., Chung, D.D.L. (2002). Increasing the electromagnetic interference shielding effectiveness of carbon fiber polymer-matrix composite by using activated carbon fibers. Carbon, 40(3): 445-447. https://doi.org/10.1016/S0008-6223(01)00133-6

[2] Wang, B., Guo, Z., Han, Y., Zhang, T. (2013). Electromagnetic wave absorbing properties of multiwalled carbon nanotube/cement composites. Construction and Building Materials, 46: 98-103. https://doi.org/10.1016/j.conbuildmat.2013.04.006

[3] Dai, Y., Sun, M., Liu, C., Li, Z. (2010). Electromagnetic wave absorbing characteristics of carbon black cementbased composites. Cement and Concrete Composites, 32(7): https://doi.org/10.1016/j.cemconcomp.2010.03.009

[4] Qiu, J.J., Guan, B., Ding, D., Zhang, H. (2015). Research on electromagnetic wave absorption properties of graphite-mixed cement-based composites. Journal of East China Jiaotong University, 32(3): 103-107,132. https://doi.org/10.16749/j.cnki.jecjtu.2015.03.014

[5] Lv, S.Z., Chen, N., Wang, H.B., Huo, J.C. (2010). Electromagnetic wave absorption performance of cement-based composites doped with ferrite and graphite. Journal of Composite Materials, 27(5): 73-78. https://doi.org/10.1016/S1872-2040(09)60084-0

[6] Li, G.Y., Yang, J., Zeng, L.B., Zhao, X.H., Xu, S.L. (2013). Smart properties of SBR emulsion modified carbon nanotubes cement mortar. Journal of Building Materials, 16(1): 65-69. https://doi.org/10.3969/j.issn.1007-9629.2013.01.012

[7] Xiong, G., Deng, M., Xu, L., Tang, M. (2004). The microwave absorbing properties of cement-based composites. Journal of The Chinese Ceramic Society, (10): 1281-1284. https://doi.org/10.1007/BF02911033

[8] Guo, A.L., Gao, R., Ba, H.L. (2013). The electromagnetically modified cement-based composites by coating and absorbing properties. Journal of South China University of Technology (Natural Science Edition), 41(2): 135-140. https://doi.org/10.3969/j.issn.1000-565X.2013.02.022

[9] Xu, L.M., Tao, M., Nie, P.C. (2015). Research on cement-based microwave absorbing materials containing EPS and carbon black. Journal of Wuhan University: Engineering Edition, 48(2): 5 . https://doi.org/10.14188/j.1671-8844.2015-02-008

[10] Zhao, Q.X., Zhang, J.R., Zhao, R.R. (2011). Influence and mechanism of carbon black content on microwave absorption properties of cement-based materials. Journal of the Chinese Ceramic Society, 39(12): 2013-2020. https://doi.org/10.14062/j.issn.0454-5648.2011.12.011 